\title{
Wind Frame: An Interactive Sonic Installation, for Listening From Wind Flow
}

\author{
Eunjoo Shin \\ Interactive Design, College of Fine Art and Design, Incheon Catholic University \\ 12, Haesongro, Yeonsu-gu, Incheon 406-840, Korea \\ ejoo@iccu.ac.kr
}

\begin{abstract}
This paper presents Wind Frame, a sonic display and machine that can listen from wind flow. The installation is based on interactive, reactive, and generative processes as they relate to intersections between natural and mechanical systems. It develops the sonification of wind flow, in a series of interactive projects to re-generate a natural phenomenon through a combination of analog and digital technology. The interaction technique involves the use of an accelerometer, Bluetooth modules, ventilation fans, relays, an Arduino processor, and a mechanical structure for generating and amplifying artificial wind sounds. This work investigates the qualities of audible and tactile display, mechanical device and interactive sound art, while addressing multi-sensory aspects of air movement.
\end{abstract}

Keywords: Interactive installation, sonic display, artificial wind, interactive sound art, ventilation fan

\section{Introduction}

Interactive computing technologies have been able to create artificial environments, which suggest new communication styles and interpretations of our perceptional reaction to the existing environment. As more of our daily life and living spaces is get involved in technology, interactive art and design works, including artificially built environments, interactive art using weather information, and creating artificial natural elements for automatic planting, are being developed. This study is an inspiration of designing a digital-physical interaction within an artificially built environment and exploring the development of a sonic interactive installation that can emphasize the change of natural wind.

Wind Frame is a sonic display, a machine listening from wind flow. This installation explores the qualities of audible and tactile display, mechanical device and interactive sound art, while addressing multi-sensory aspects of air movement. It is based on the idea of designing a system to convey a subtle perception of changes in wind breeze in an environment by concentrating the sonic and tactile perception of an artificial airflow, which is transferring changes in the wind breeze outside the gallery

The installation has been developed using conceptual and technical interactive designs as follows: (1) appropriate fans as a medium for delivering air movement; (2) an audible and tactile display; and (3) a sonic device that balances the use of analog and digital elements to generate artificial wind sounds.

The remainder of this paper is structured as follows. It begins with reviewing related works in the context of a sonic installation that uses fans. Next, the mechanical components of the installation, its implementation process, and the interactive sound that Wind Frame provides are described. Finally, it concludes by framing the direction I would like my future work to take. 


\section{Related Work}

The stimulation of multiple human senses initiates a feeling of presence as well as of the environment that we consider as a part of our life. Wind is one of the natural elements, which we are not aware of visually but through subtle sonic and tactile effects. So fans, an analog medium has often been used for generating audible sound and tactile airflow in art installations without visual effects.

In the following section, art works on the perception of wind are presented including 30,'000 Plastic bags, 16 ventilators, Blow Up and FLOW 5.0 by Zimoun, Scott, and Studio Roosegaarde. In these art works, AC ventilation fans and computer fans are manipulated to produce discrete airflow as well as artificial air moving sounds.

\subsection{0'000 Plastic Bags, 16 Ventilators}

30 '000 Plastic bags, 16 ventilators explores mechanical airflow to transform and activate a space. It was created by Zimoun, an artist known for sound and architectural scaled-installations. This work combines visual, sonic, and spatial elements as an architectural intervention of sound using a simple mechanical system.

It consists of 30,000 plastic bags on a wall and 16 ventilation fans on a ceiling, facing toward them. The white plastic bags cover a wall and by artificial air from the ventilation fans, they create an acoustic hum similar to natural phenomena such as snow blows. This art work similarly develops its own behavior and rules by arranging industrially produced parts according to seemingly simple rules and using the physicality of these industrial materials without any digitized controls.

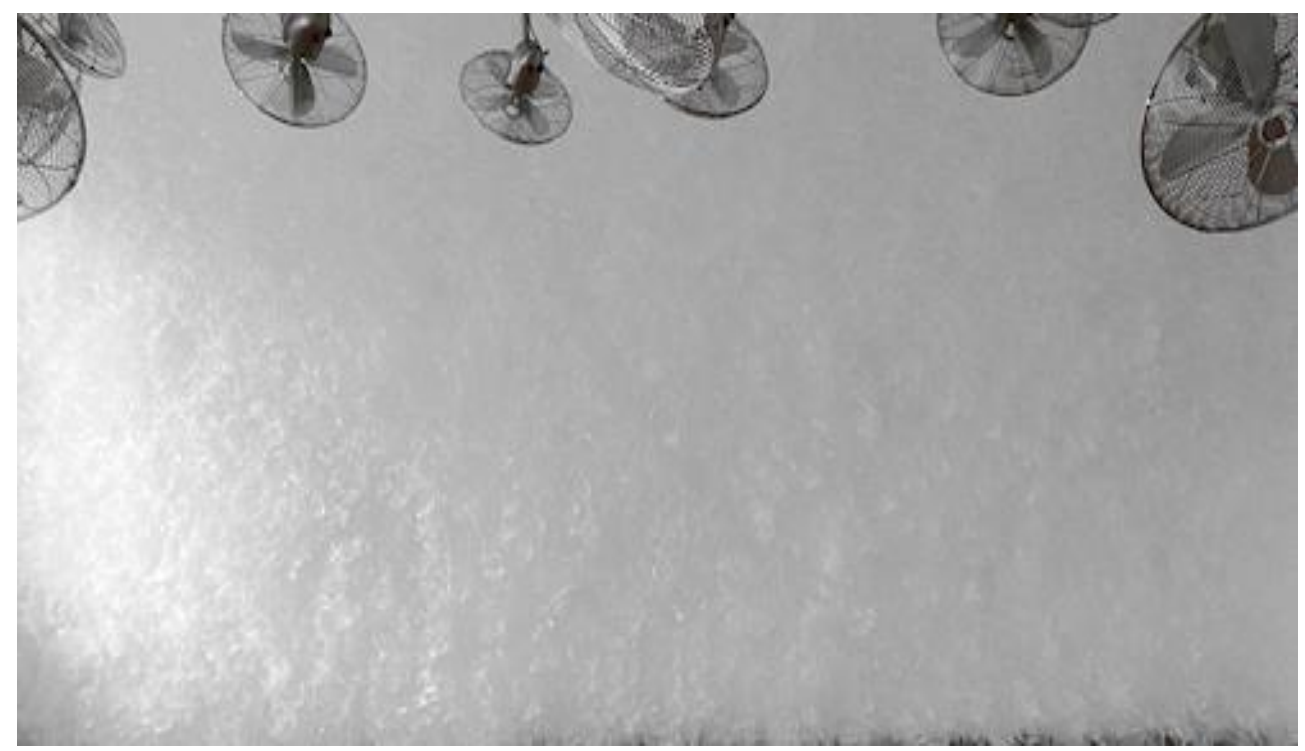

Figure 1. 30'000 Plastic Bags, 16 Ventilators, 2010

\subsection{Blow Up}

Created by interactive artist Scott Snibbe, Blow Up is an interactive sculpture installed at the Yerba Buena Center for the Arts in San Francisco. Blow Up simultaneously generates processes of recording, translation, and amplification to increase the breath's legibility, while detaching the breath from the body.

It is composed of two parts: 12 small impellers on one side of the room and a wall of 12 large fans on the other side of the room. The first part, a rectangular array of 12 small impellers stands on a table on one side of the gallery. This small input device is electronically linked to the second, a large wall of 12 electric fans. The array of small 
impellers is spatially and temporally synchronized to the fans on the wall. It records and transmits a pattern of human breath to a room-sized filled of wind powered wall of large electric fans. The wall of large fans continues to play back amplified wind patterns in real-time and the system stores the most recently captured breath patterns and replays them in a loop until someone breathes a different pattern. The process of observing this translation and trans-location of respiratory activity may prompt the sender to consider the connection between one's person and the air he exchanges.

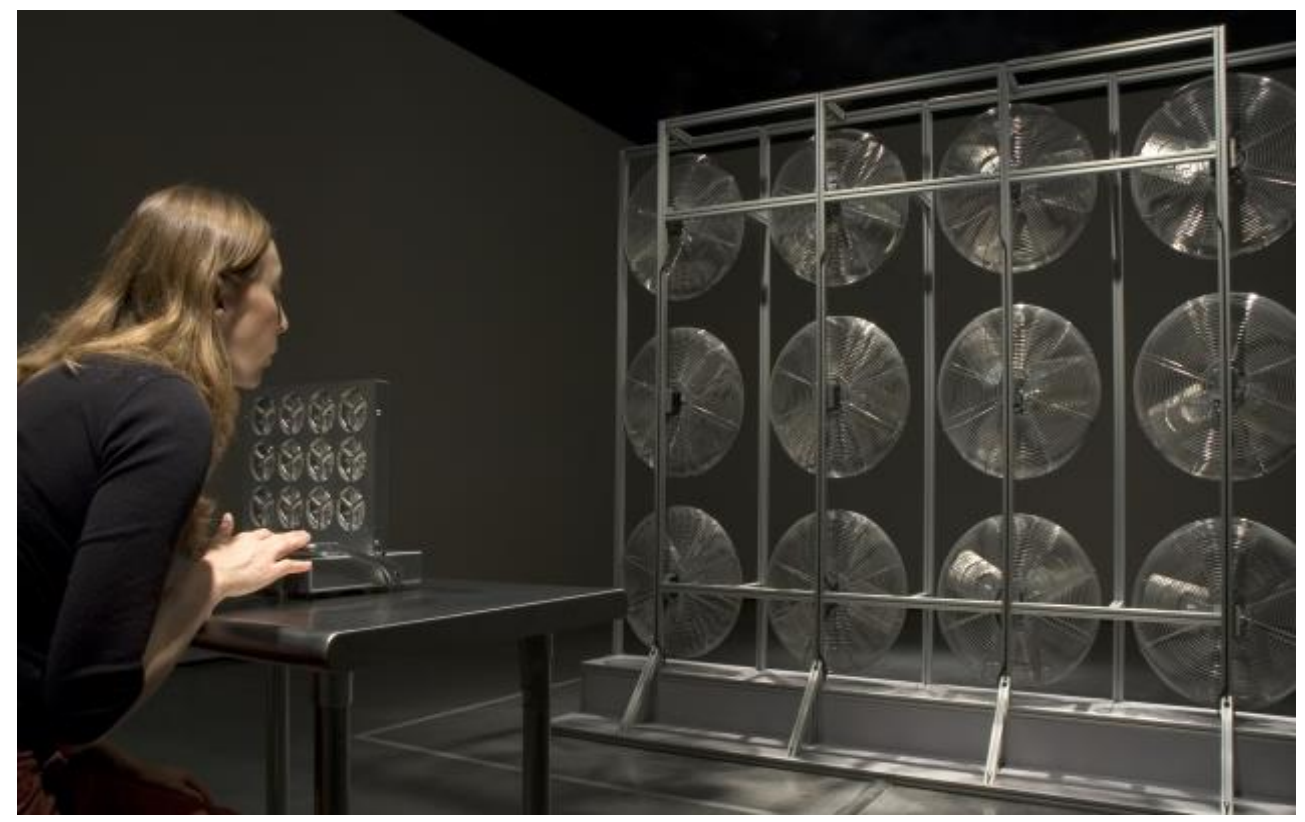

Figure 2. Blow Up, 2010

\subsection{Flow 5.0}

Flow 5.0, awarded the Dutch Design Award in the category of Best Autonomous Spatial Design, is officially a part of the 21c Museum Art Collection USA. It is a large smart wall that reacts to the sound and motion of audience passing by combining human senses and technology. This installation is a modular system of several meters, $50 \mathrm{~cm}$ in length, $100 \mathrm{~cm}$ in width, and $200 \mathrm{~cm}$ in height and consists of hundreds of ventilators, aluminum, sensors, microphones, electronics, and software. In essence, the fans controlled by sensors adjust its direction and speed based on the audience's movements around them.

As the audience approach, sensors located within the installation activate the fans, which blow air onto passersby and into the surrounding space. By walking, speaking, and interacting with its surface, the audience triggers the emergence of an elusive landscape created out of transparent fields and artificial wind. The site specific installation, Flow 5.0 heightens the audience's consciousness as a collective body while actually becoming a dynamic relationship between space and technology. Moreover, it explores a new nature, evolving from technological innovations shifting between the analog and the digital. 


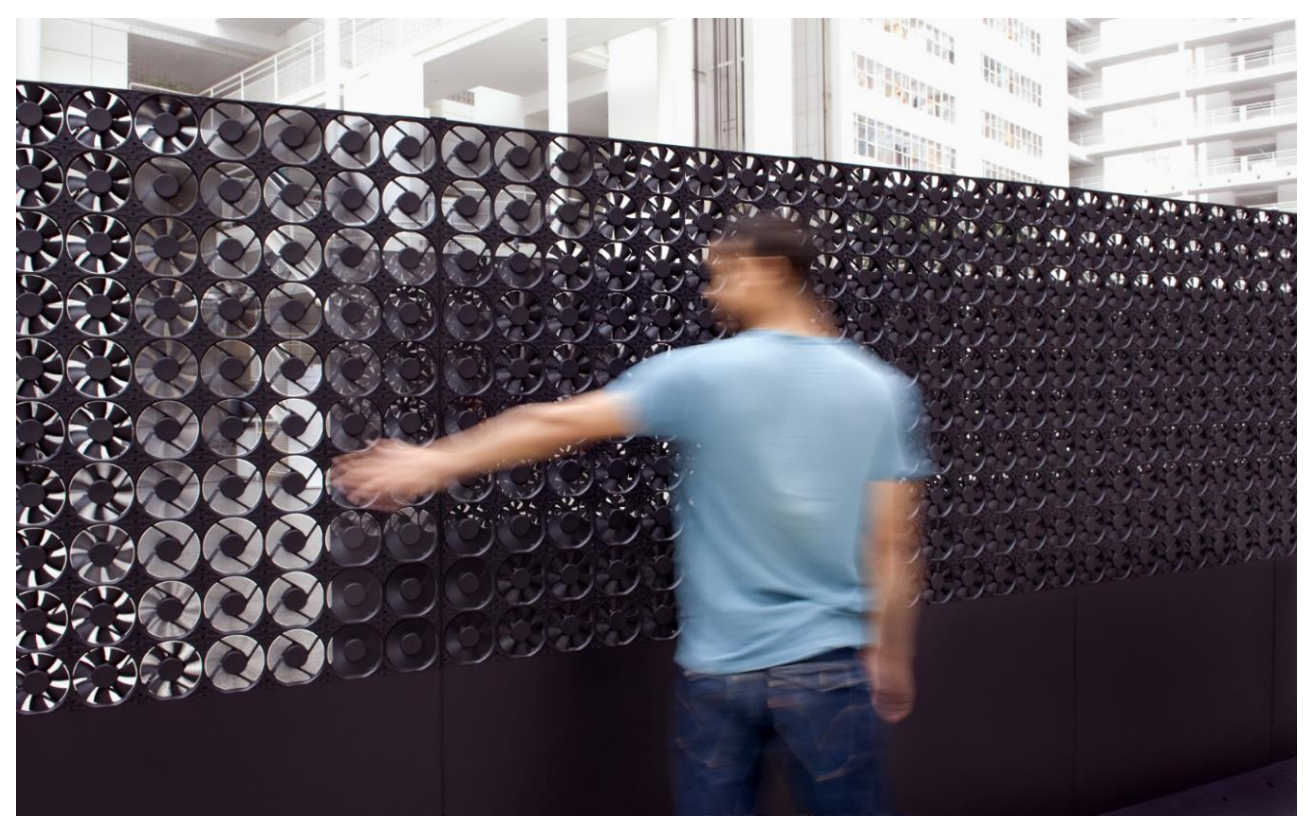

Figure 3. FLOW 5.0, 2007

\section{Interactive Installation and Technical Detail}

\subsection{Background}

Wind, as the movement of air, is one of the essential attributes of a natural environment. Wind differs from other elements of a natural environment, such as light, water, and soil, in the way that it is perceived it. It is perceived not visible but by audible and tactile senses. We become aware of it through interaction with our bodies: we hear its sound via a bulk movement of air; we feel it via the disturbance of the air near our skin. In spite of that, we may infer its existence visually through the sight of other everyday objects moving in the wind. However, we easily dismiss the indirect visual effects of wind; whereas, even if wind does not have a high velocity or energy, its movements are subtle enough to be sensed. To clearly sense a subtle wind motion, this research explores our extended experience with nature, wind in a unique way. A case study, Wind Frame is suggested to evoke serenity and emotional attachment by experiencing the meditative quality of the touch of wind artificially.

In particular, Wind Frame develops the sonification of a windy breeze, using a series of interactive projects to re-generate a natural phenomenon through a combination of analog and digital technology. The sonic content of Wind Frame collage existing wind sounds in a rectangular white frame formed by a designed mechanical structure, where the sound samples are generated and as such these simply play themselves out. The mechanical structure has the ability to collage sounds in ways that vary according to wind energy in real-time, but it does not create a direct relationship between the quality of an audience's gesture and the sonic outcome. In addition to that, it explores the synergy between an immersive experience based on a natural environment and sonic and tactile senses.

\subsection{Configuration}

Wind Frame is a sonic display and interactive installation concerning interactive, reactive, and generative processes as it relates to intersections between natural and mechanical systems. It translates wind flows from outside to artificial wind sounds produced by ventilation fans in a white metal frame that looks like a simple painting. Its mechanical system is set in motion to create sonic drawings and movements by capturing 
subtle changes in wind with respect to space and time.

Wind Frame consists of one large white metal frame that is built in with 28 ventilation fans, 28 relays, a computer programmed controller, and a separate small tilting device that includes an accelerometer. The metal frame installed in the gallery and the tilting device installed outside can communicate through Bluetooth modules. It listens to the wind flow data from the tilting device and reproduces moving wind sounds through circle-lined ventilation fans by varying time and amplitude. This outcome plays the role of a mediator, providing a mechanical perspective to subtle natural changes.

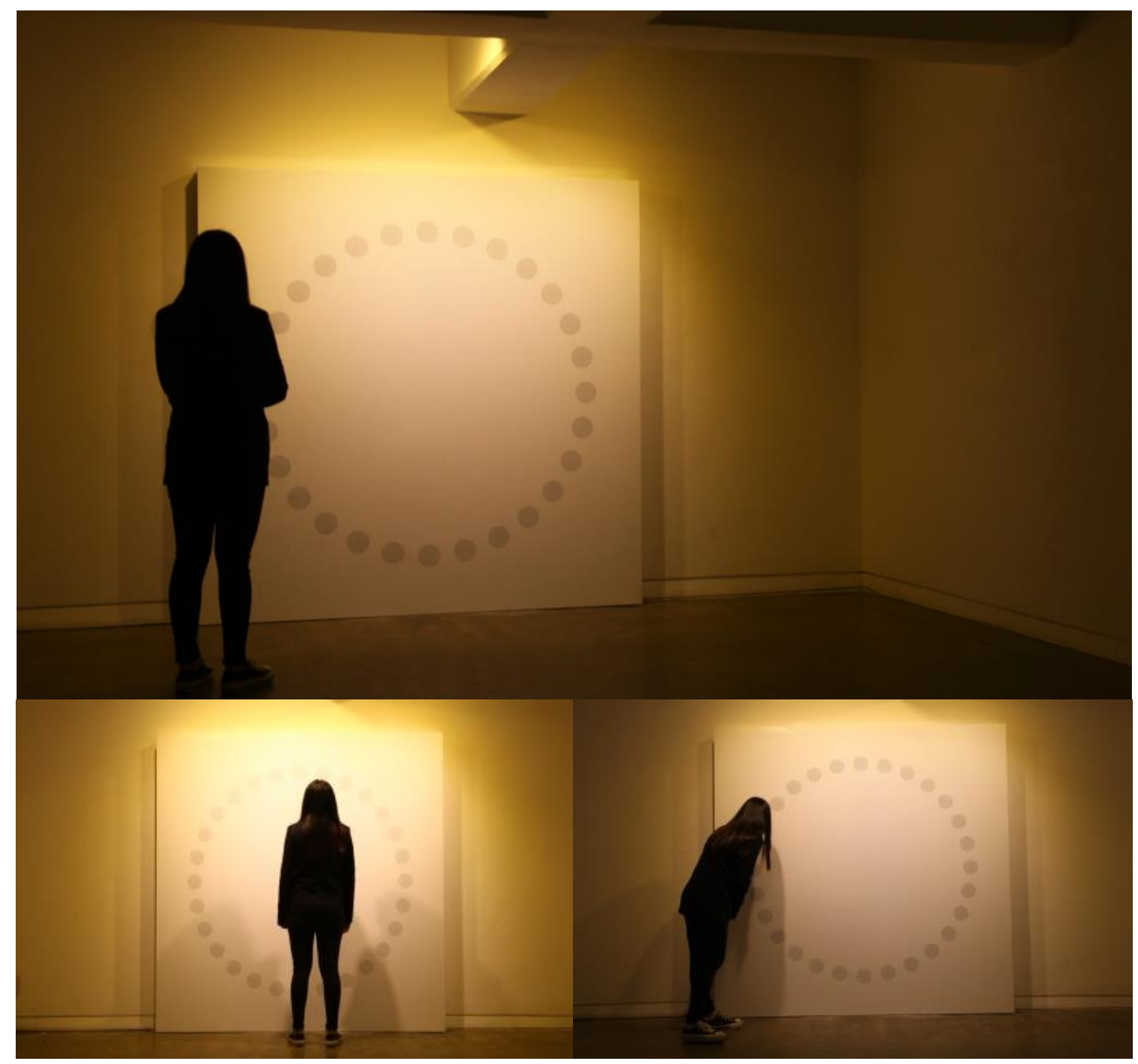

Figure 4. Wind Frame, 2013

\subsection{Structure}

To create a hardware system with the ability to simulate wind in an indoor space, one has to consider certain factors, such as real-time operation and the quality of sound. The fabricated system meets the requirements of wind simulations generating amplified sounds of the wind from the outside on a real-time basis.

3.3.1. Physical Structure: The wind frame consists of three parts: (1) a metal frame, (2) a donut shaped acrylic panel, and (3) 28-AC-powered fans. The first part is a white metal frame with a dimension of 2,000 mm (w) x 2,000 mm (l) x $200 \mathrm{~mm}(\mathrm{~h})$ and it stands against a wall in the gallery. In front of the frame, there are several small holes to let artificially blowing wind and sounds go out. The back of the frame is left open for assembling the rest of the parts. The second part is an acrylic ring that is adhesed on the back of the frame using silicon. The ring has 28 holes to hold ventilation fans and relays. On a bar across the diameter of this ring, a computer 
programmed Arduino processor is attached to control fans and relays. The ACpowered fans and relays are arranged in a circle array, and here, the front of each fan faces toward an acrylic ring.
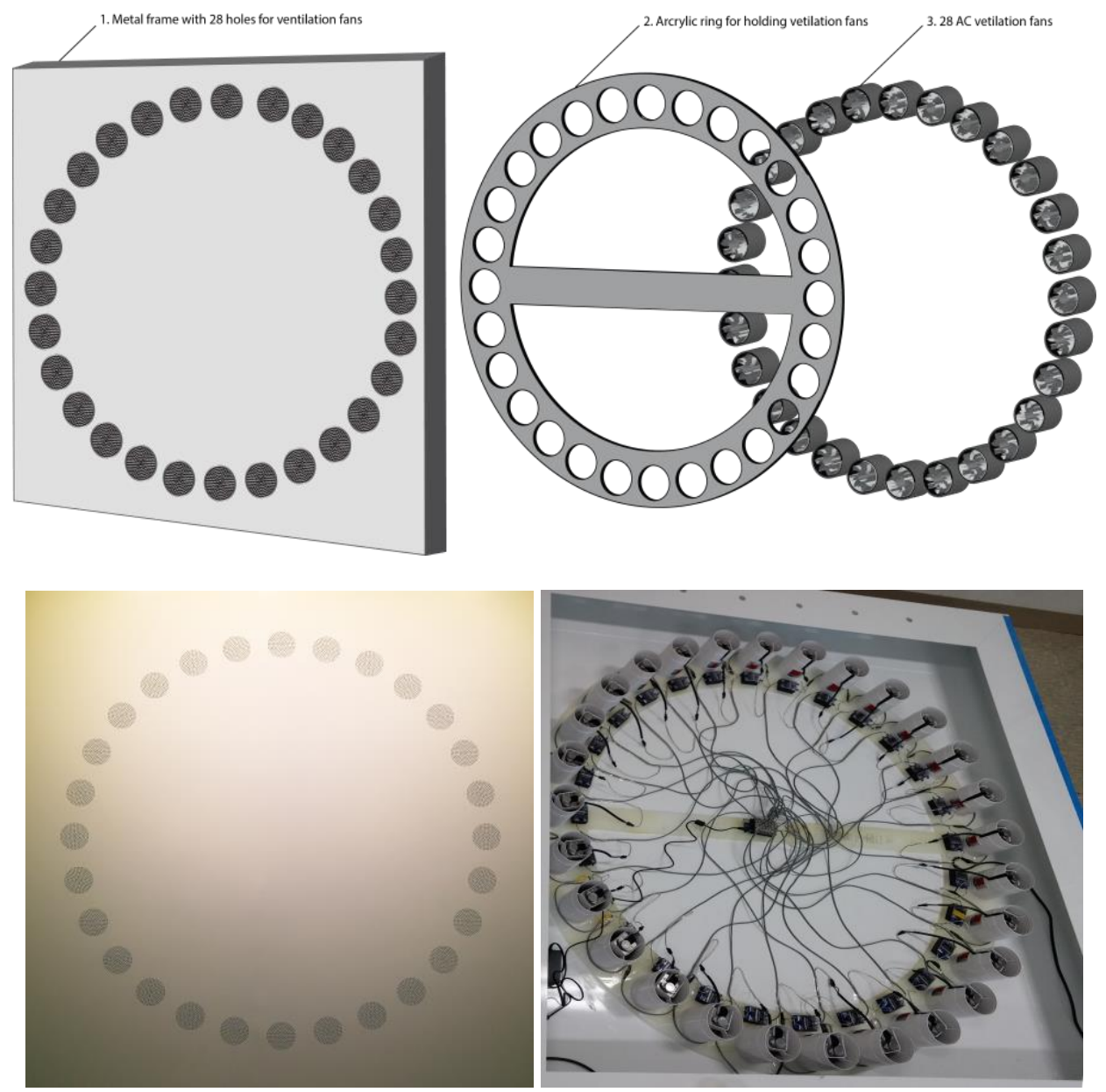

Figure 5. The Physical Structure

3.3.2. Sound Amplifying Structure: Manufactured 220_V AC-powered fans are fabricated for better quality of wind blowing sound. To amplify blowing sounds, a zinc plate was used to cover three-quarters of the front in each ventilation fan. This forces the generated air to hit the surface of the plate, and so the vibrations caused by this impact are amplified physically. A soft speaker cloth is placed on the top of the zinc plate. In general, its main purpose is to protect the internal elements of a speaker and also to allow the sound to pass through clearly. Because it is situated directly on the vibrating zinc plate, it dampens the rattling noises and also protects the fans from small, lightweight obstacles. To reduce the noise of the fan motors, one-third of the back of each ventilation fan is covered too; however, the back of the fans is not entirely covered to allow cooling of the heat generated by the motors. The ventilation fans of the installation produces quiet wind sounds, and therefore, must be installed in an isolated space away from loud-sound installations. 

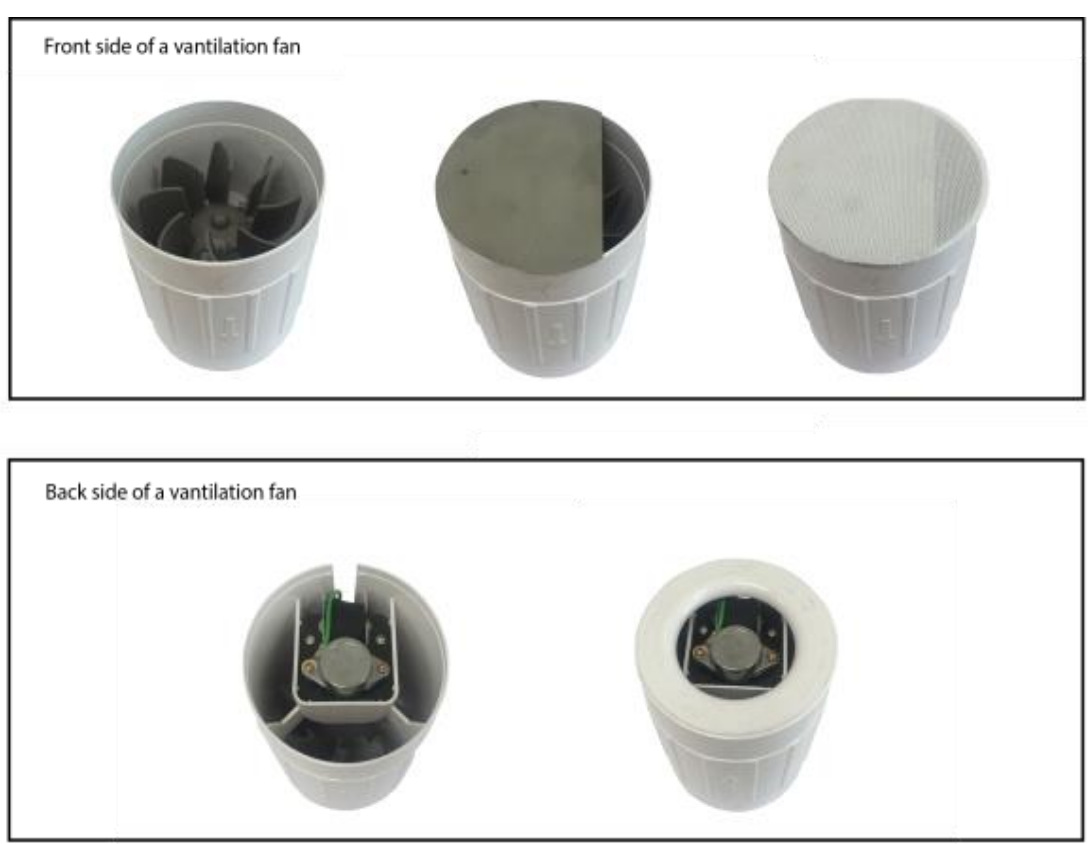

Figure 7. The Sound Amplifying Structure

\subsection{Technical Operation}

In addition to developing the physical aspects of the structure, the mechanical aspects in terms of simulation and control have also been developed. As shown in the schematic in Figure 6, a mechanism for controlling the input part to detect the wind blowing from the outside and an output part to generate artificial wind from a physical system was organized.

The tilting device outside the gallery contains a flexible panel connected to an accelerometer, a Bluetooth module, and an Arduino processor. All the electronic components excluding the accelerometer are enclosed in a small box. The accelerometer, installed in the flexible panel, detects the movement of wind flow and this is digitalized by an Arduino processor. Subsequently, a Bluetooth module transmits the data of the accelerometer to another Bluetooth module in the metal frame located inside the gallery.

The received data is analyzed by another Arduino processor. It is used to control the speed of the ventilation fans by turning these on or off via relays that operate as electrical switches. The ventilation fans in the gallery create artificial and amplified wind blowing sounds in real-time. The fans spin at different speeds based on the input information on the wind flow outside. The electronic elements are digitally operated to generate artificial wind, but the created audible sound and tactile air flow are a quiet, analog-typed transmission. 


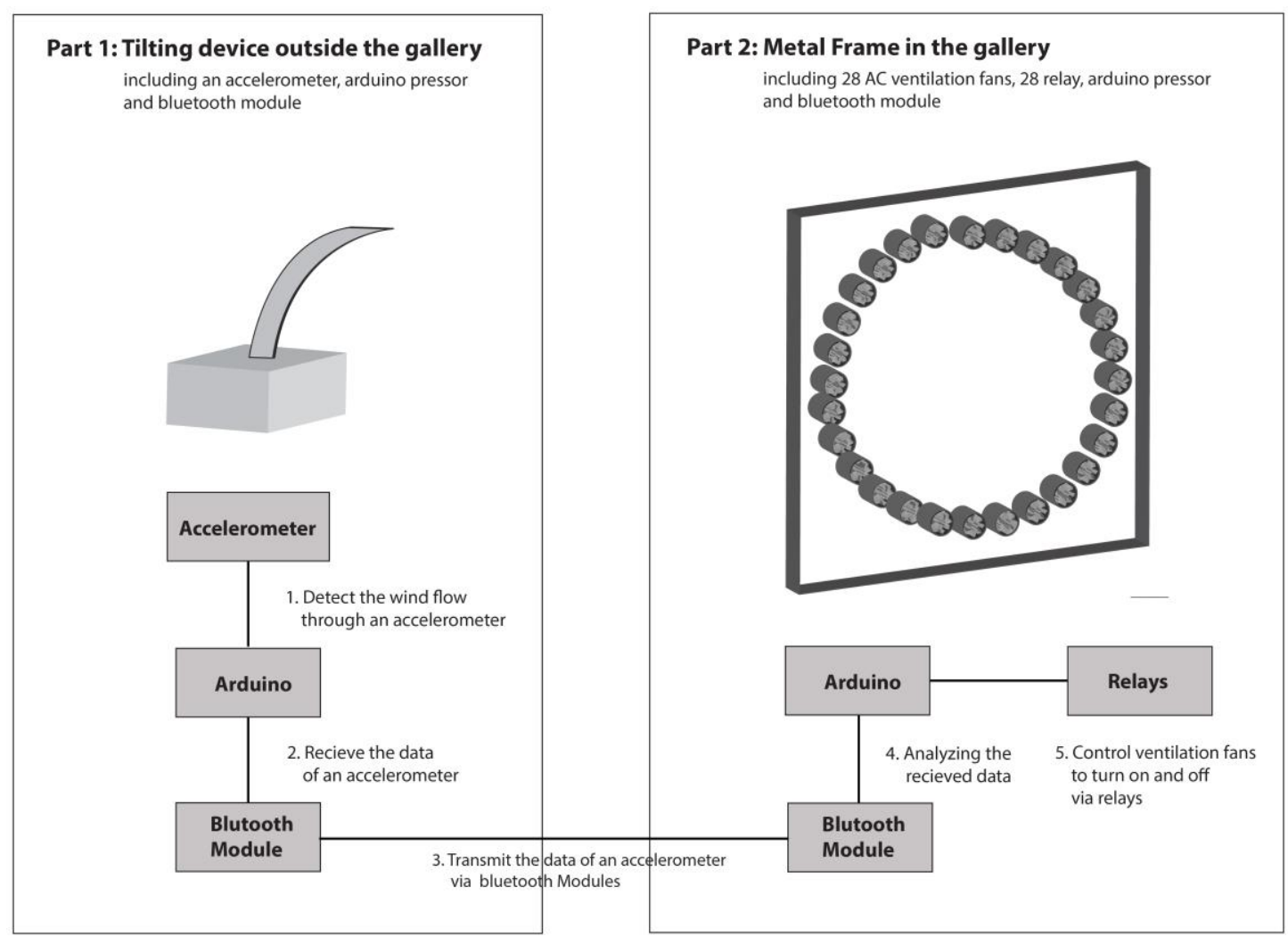

Figure 6. Technical Diagram

\section{Conclusions and Future Work}

In this work, the installation is based on the idea of trapping natural sound in a frame like a painting that can be appreciated visually, but the developed device-based frame emphasizes the multi-sensory, audio and tactile aspect rather than the visual aspect. This work is also developed to focus on an interactive installation interpreting sound, which is an interaction between a mechanical system and natural wind monitored by a digital process. For the interaction, the information from the accelerometer that relates to the force of wind outside, is used to simulate a wind sound generated by ventilation fans in a frame inside. The sound texture varies with the data on the wind force in real-time and is amplified in the inner space of a large metal frame.

Furthermore, Wind Frame challenges audiences to understand a new way of sensing wind that is not in the immediate environment. It allows audiences to concentrate on feeling the natural phenomenon, of wind more audibly and sensibly within the artificially built space. It does not offer direct audience interaction between the installation and wind, and so it can be experienced more like a kinetic piece. The sound moving effect produced through implicit mechanisms and the space around Wind Frame was transformed leaving a subtle experience of wind energy, when it was presented at the exhibition, RESONATE: Media Spaces and Installations at Gallery Palais de Seoul.

Wind Frame was a sonic display and machine listening from wind flow and creating a communication space by manufacturing multi-sensing media with an emotional intention. The audience exchanges feedback with the work through an emotional and sensatory experience rather than a dynamic physical participation. In the future, I will try to add other interaction such as networking which would let audiences choose a specific location for perceiving wind flow through the artwork. I will also improve on the quality of the amplified artificial wind sound generated by analog fans as well as filter fan motor's noises. 


\section{Acknowledgements}

This research was supported by the Incheon Catholic University Research Grant of 2015.

And also the art work presented as a case study in this research was partly funded by Artist Support Program on Human-centered Interaction by Red Pool, a media company in Korea.

\section{References}

[1] D. Rocchesso, P. Polotti and S. D. Monache, S. D, J. Design, vol. 3, no. 13, (2009).

[2] E. Shin and H. Yoo, J. Multimedia U. Engineering, vol. 10.2, no. 405, (2015).

[3] K. Franinović and S. Serafin, Editors, "Sonic Interaction Design", Mit Press, Cambridge, MA, (2013).

[4] N. D. Oliveira and N. Oxely, Editors, "Installation Art", Thames and Hudson, London, (1994).

[5] A. Rydarowski, O. Samanci and A. Mazalek, Editors, "Murmur: kinetic relief sculpture, multi-sensory display, listening machine", Proceeding of the 2nd international conference on Tangible and embedded interaction, (2008) February 18-20, Bonn, Germany.

[6] A. V. Moere and N. Woulters, Editors, "The Role of Context in Media Architecture", Proceedings of the 2012 International Symposium on Pervasive Displays, (2012) June 4-5, Proto, Portugal.

[7] F. Hülsmann, J. Fröhlich, N. Mattar and I. Wachsmuth, Editors, "Wind and warmth in virtual reality: implementation and evaluation", Proceedings of the 2014 Virtual Reality International Conference, (2014) April 09-11, Laval, France.

[8] A. Sherstyuk, Editor, "Haptic wind", Proceedings of the 2008 International Conference on Advances in Computer Entertainment Technology, (2008) December 3-5, Yokohama, Japan.

[9] H. Ishii, S. Ren and P. Frei, Editors, "Pinwheels: visualizing information flow in architectural space", Proceedings of CHI '01 Extended Abstracts on Human Factors in Computing Systems, (2001) March 31-April 5, Seattle, USA.

[10] E. J Shin, Editor, "Sonic Display and Interactive Machine, Listening from Wind Flow", Proceedings of 2015 International workshop on Art, Culture, Game, Graphics, Broadcasting and Digital Contents, (2015) August 19-22, Jeju Island, Korea.

[11] D. Bowen, "in Tele-present wind", Leonardo, vol. 44, (2011) August 7.

[12] "Arduino", http://arduino.cc.

[13] “Zimoun", http://www.zimoun.net/2010-30000.html.

[14] S. Snibbe, http://www.snibbe.com/digital-art\#/projects/interactive/blowup.

[15] "Studio Roosegaarde", https://www.studioroosegaarde.net/project/flow/photo/\#flow-5-0.

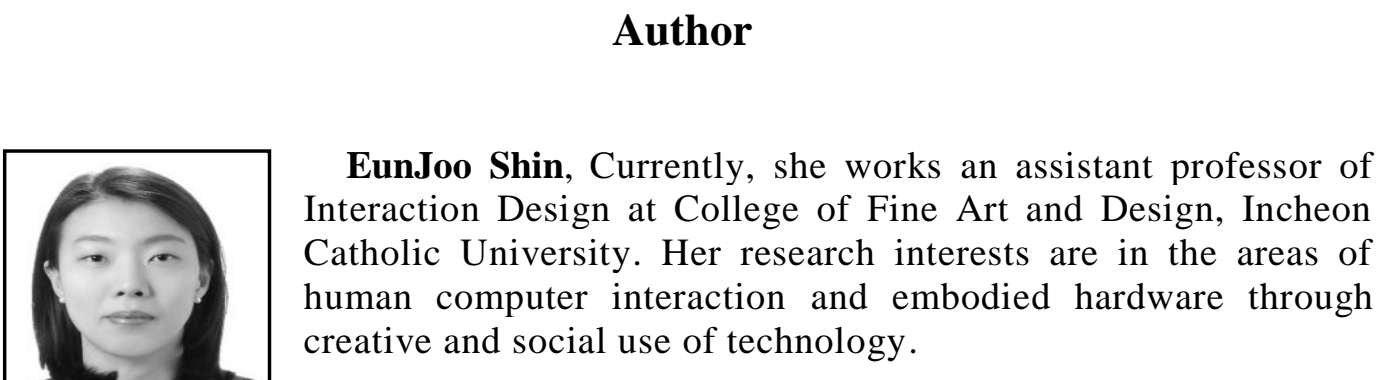


International Journal of $u-$ and e- Service, Science and Technology Vol.8, No. 9 (2015) 